\title{
Praticando o silêncio: intervenção educativa para a redução do ruído em Unidade de Terapia Intensiva
}

\author{
Practicing silence: educational intervention for reducing noise in the Intensive Care Unit
}

Practicando el silencio: intervención educativa en la reducción del ruido en la Unidad de Cuidados Intensivos

\section{Silvana Triló Duarte', Maiara Matos', Tatiane Cristina Tozo", Luis Carlos Toso", Aline Aparecida Tomiasi', Péricles Almeida Delfino Duarte"}

' Faculdade Assis Gurgacz, Curso de Fonoaudiologia. Cascavel-PR, Brasil.

"Faculdade Assis Gurgacz, Hospital São Lucas. Cascavel-PR, Brasil.

\author{
Submissão: 31-01-2011 Aprovação: 21-05-2012
}

\begin{abstract}
RESUMO
O objetivo deste estudo foi avaliar se os níveis de pressão sonora dentro da UTI são diminuídos após intervenção educativa com a equipe multiprofissional. Foram mensurados os níveis de ruído no interior da UTI (através de um decibelímetro instalado próximo à cabeceira de um paciente) durante sete dias, sendo repetido o procedimento após uma intervenção educativa, a qual consistiu de palestras, cartazes e dramatizações, entre outros. Houve grande redução do nível de ruído entre o período pré e pós-intervenção, em todos os horários avaliados. As principais fontes de ruídos dentro da UTI foram da própria equipe. Os níveis de ruído encontrados estiveram acima do recomendado. O estudo mostrou que, com uma intervenção educacional junto à equipe da UTI e sua conscientização sobre os mecanismos e efeitos, é possível haver redução dos níveis de ruído e consequente eestressee do ambiente.
\end{abstract}

Descritores: Ruído; Ruído ocupacional; Educação; Unidades de Terapia Intensiva.

\begin{abstract}
The objective of this study was to evaluate whether the sound pressure levels are decreased in the ICU after an educational intervention with the multidisciplinary team. Noise levels were measured inside the ICU (using a decibelimeter installed near the bedside of a patient) for seven days, and repeated the procedure after an educational intervention, which consisted of lectures, posters and dramatizations, among others. There was a large reduction in noise level between the pre and postintervention period, at all times evaluated. The main sources of noise in the ICU were the own team. The noise levels were higher than recommended. The study showed that with an educational intervention with the ICU staff and their awareness of the mechanisms and effects, it is possible to have reduced levels of noise and consequent estresse environment.
\end{abstract}

Key words: Noise; Noise, occupational; Education; Intensive Care Units.

\section{RESUMEN}

El objetivo de este estudio fue evaluar si los niveles de presión sonora se redujeron en la UCI después de una intervención educativa con el equipo multidisciplinario. Se midieron los niveles de ruido en la UCl (utilizando un decibelímetro instalado cerca de la cabecera de un paciente) durante siete días, y se repite el procedimiento después de una intervención educativa con el equipo, que consistió en conferencias, carteles y dramatizaciones, entre otros. Hubo una gran reducción en el nivel de ruido entre el período pre y post-intervención, en todos los tiempos evaluados. Las principales fuentes de ruido en la UCI fueron del proprio equipo. Los niveles de ruidos encontrados eran más altos que los recomendados. Este estudio mostró que con una intervención educativa y de sensibilización acerca de los mecanismos y los efectos del ruido con el personal de la UCl, es posible lograr la reducción de los niveles de ruido y el estrés ambiental consecuente.

Palabras clave: Ruido; Ruido en el ambiente de trabajo; Educación; Unidades de Terapia Intensiva. 


\section{INTRODUÇÃO}

O ambiente da Unidade de Terapia Intensiva (UTI) tem características potencialmente danosas tanto aos pacientes e familiares quanto à equipe. Este ambiente hostil pode gerar consequências a curto e longo prazo, como estresse, delirium, burnout e síndrome de estresse pós-traumático ${ }^{(1)}$. Entre os principais componentes ambientais, o ruído é um dos mais marcantes ${ }^{(2)}$. Têm-se detectado níveis altos de ruído em diversos ambientes hospitalares, como Pronto-Socorro, Centro Cirúrgico e Enfermarias ${ }^{(3)}$, porém as UTIs fornecem um ambiente único para que o excesso e permanência de ruído possam ser prejudiciais ${ }^{(4)}$.

O ruído é caracterizado por diferentes sons com diferentes frequências, que causam efeitos inesperados às pessoas expostas a ele, prejudicando sua saúde ${ }^{(5)}$. Ou seja, é um som indesejável, desagradável e potencialmente perigoso à saúde ${ }^{(2)}$.

A Organização Mundial de Saúde (OMS) reconheceu que o ruído pode causar danos à saúde das pessoas expostas a ele, como: perturbar o trabalho, o descanso, o sono, a comunicação dos seres humanos, prejudicar a audição e causar reações psicológicas, fisiológicas e patológicas nos indivíduos expostos ${ }^{(6)}$. Entre vários danos fisiológicos, o ruído pode provocar distúrbios cardiovasculares, redução da saturação arterial de oxigênio, perda auditiva, aumento de secreção gástrica, estimulação da hipófise e adrenal, alteração do sono fisiológico, imunossupressão e redução da cicatrização( ${ }^{(7)}$.

Vários estudos têm demonstrado a frequência e importância do ruído no ambiente da UTI(8), embora a maioria dos estudos seja em UTIs neonatais ou pediátricas ${ }^{(1)}$. Por outro lado, menos tem sido estudado sobre o impacto de intervenções educativas sobre o nível de ruído neste ambiente ${ }^{(9-10)}$.

\section{OBJETIVO}

O objetivo deste estudo foi verificar o nível de pressão sonora dentro de uma Unidade de Terapia Intensiva geral, em diferentes horários, e avaliar o impacto de uma intervenção educativa junto à equipe, na redução destes níveis sonoros. Esta intervenção tinha como foco o ruído, visando a uma mudança de comportamento dos profissionais, favorecendo a qualidade de vida da equipe multiprofissional, assim como os pacientes internados.

\section{MATERIAIS E MÉTODOS}

Tratou-se de um estudo de intervenção, não randomizado, não cego, controlado (o próprio grupo serviu como controle), com avaliação antes e depois da intervenção.

O estudo foi realizado em uma UTI Geral (clínica, cirúrgica, cardiológica e trauma) que atende pacientes adultos e pediátricos (acima de 30 dias de vida) de um hospital privado universitário de 150 leitos na Cidade de Cascavel (estado do Paraná). Essa UTI tem capacidade para 20 leitos, tendo cerca de $1000 \mathrm{~m}^{2}$ de área. Tem no seu quadro de trabalho médicos, enfermeiros, técnicos de enfermagem, fisioterapeutas, secretária, escriturária, psicóloga, assistente de farmácia e equipe de limpeza. Conta ainda com vários profissionais que transitam nesse setor, como outros médicos assistentes, profissionais de radiologia e laboratório, equipe de nutrição, equipe de controle de infecção hospitalar, de fonoaudiologia, de manutenção, além de estudantes de várias áreas da saúde. O turno de trabalho é dividido em manhã, tarde e noite. A relação profissional de enfermagem / paciente é de 1:2, sendo que há duas enfermeiras supervisoras por turno. Em cada turno há dois médicos, duas enfermeiras, cinco a sete técnicos de enfermagem e uma fisioterapeuta de plantão, totalizando de dez a doze profissionais fixos na UTI em cada turno. A passagem de plantão entre os profissionais costuma ser à beira do leito. As rotinas de visitas de familiares na UTI são de 3 a 5 visitas por dia (cerca de 15 minutos cada); além disso, os pacientes conscientes, ou em desmame de VM, têm acompanhantes por cerca de 12 horas por dia.

O protocolo de pesquisa foi aprovado pelo Comitê de Ética da Faculdade Assis Gurgacz, 089/2010 (Ata 007/10), tendo sido dispensado Termo de Consentimento aos participantes.

Para a realização da pesquisa, foi utilizado um decibelímetro (aparelho que mede o nível de pressão sonora em decibéis níveis de audição $[\mathrm{dB}(\mathrm{A})]$ da marca Impac ${ }^{\oplus}$ modelo IP-410 (São Paulo, Brasil). Sua classe de precisão é de $\pm 3,5 \mathrm{~dB}(\mathrm{~A})$. A escala que foi utilizada para a medição do nível de pressão sonora na UTI foi de 30 a $100 \mathrm{~dB}(\mathrm{~A})$. O estudo foi realizado em três etapas.

\section{Primeira etapa}

Foram coletados os níveis de ruído dentro da UTI com o aparelho decibelímetro, posicionado a cerca de um metro de distância da cabeça dos pacientes. Foram estipulados três intervalos de tempo para a quantificação dos níveis de ruído: das $11 \mathrm{~h}$ às $11 \mathrm{~h} 29$, das $12 \mathrm{~h} 45$ às $13 \mathrm{~h} 14 \mathrm{e}$ das $23 \mathrm{~h}$ às $23 \mathrm{~h} 29$. Estes três horários foram escolhidos por representar momentos distintos da rotina da UTI: o primeiro $(11 \mathrm{~h}$ às $11 \mathrm{~h} 29)$ corresponde à visita de familiares; o segundo (12h45 às 13h14) corresponde a troca de plantão da equipe; e o terceiro (23h às $23 \mathrm{~h} 29)$ tenta representar um momento mais calmo. Os dados foram coletados de minuto a minuto, totalizando 30 dados diferentes por cada intervalo. Esse procedimento foi realizado diariamente, em um período de sete dias.

A coleta do nível de pressão sonora foi realizada por uma acadêmica de Fonoaudiologia (PG), previamente treinada. Ela se posicionava em pé, ao lado do leito (no interior do box), a cerca de um metro da cabeça do paciente, portando o decibelímetro. O equipamento apresentava o valor em um display, medindo de maneira contínua, mas sem registrá-lo. Durante cada minuto era anotado pelo pesquisador o valor mais alto neste período, bem como quaisquer eventos ou acontecimentos que pudessem ser relevantes (como, por exemplo, procedimentos no paciente, alarmes, intercorrências clínicas, admissão, telefone tocando, etc). Era feito um rodízio aleatório entre os leitos, sendo um leito (box) a cada dia, embora o leito pudesse ser repetido.

Essa primeira etapa aconteceu em caráter confidencial a fim de se preservar a rotina dos profissionais e não haver interferência nos resultados de coleta. Assim, apenas a chefia médica e de enfermagem da UTI tinham conhecimento do procedimento. Os outros profissionais foram informados que se tratava de procedimento de coleta de dados clínicos, de maneira inespecífica. 


\section{Segunda etapa}

A segunda etapa foi iniciada uma semana após a coleta inicial. Foi realizada uma intervenção educacional com os profissionais por meio de aulas expositivas e distribuição de material educativo. Nestas aulas foram abordados assuntos como: o que é ruído, qual a intensidade de ruído recomendada para hospitais, possíveis alterações que o ruído pode causar ao paciente e na equipe e quais as maneiras que esse ruído pode ser minimizado. Também foram feitas nessas apresentações simulações do ambiente estressor e ruidoso da UTI, em que os próprios alunos simulavam ser pacientes enquanto escutavam ruídos característicos de uma UTI. As aulas foram ministradas em conjunto pelos acadêmicos, pela professora e por médico assistente da UTI.

Nessa etapa foi também feita divulgação por meio de cartazes nos ambientes da UTI sobre a importância do ruído e suas consequências, bem como estratégias sobre sua redução. $\mathrm{O}$ tempo total dessa segunda etapa foi de duas semanas.

\section{Terceira etapa}

A terceira etapa foi iniciada uma semana após o término da segunda etapa; portanto, cerca de um mês após a primeira etapa (pré-intervenção). Foi realizada a mensuração dos níveis de pressão sonora novamente, dentro da UTI, da mesma forma como foram coletados na primeira etapa, sendo realizada pelo mesmo pesquisador e com o mesmo rodízio entre os leitos. Também a coleta foi realizada durante sete dias. Nessa fase também foi tentada a realização do sigilo, porém devido à mobilização gerada pela intervenção da segunda fase (educativa), a presença do pesquisador foi identificada pela própria equipe como sendo de coleta de dados relacionados a ruído.

Todos os dados foram tabulados em banco de dados específico, utilizando-se planilha em Excel ${ }^{\circledR} 2007$. Foi realizada análise estatística com análise de pareamento de dados (Wilcoxon signed rank test) dos dados horários da coleta, e análise de variância para as médias, utilizando-se como significante $p<0,05$.

\section{RESULTADOS}

O estudo (incluindo coleta de dados e intervenção educativa) foi realizado nos meses de setembro e outubro/2010. Durante o período de estudo foram coletados dados de pressão sonora 90 vezes em cada dia, totalizando 630 medidas em cada período (primeira e terceira fase).

Tabela 1 - Pressões sonoras medidas em cada período, antes e após intervenção educativa.

\begin{tabular}{l|ccc|ccc|c}
\hline \multirow{2}{*}{ Hora } & \multicolumn{4}{|c|}{ Pré intervenção, $\mathrm{dB}$} & \multicolumn{3}{c}{ Pós intervenção, $\mathrm{dB}$} \\
& Média $\pm \mathrm{DP}$ & Mínimo & Maximo & Média $\pm \mathrm{DP}$ & Mínimo & Máximo & $\mathrm{p}$ \\
\hline $11 \mathrm{~h}$ às $11 \mathrm{~h} 29$ & $59,82 \pm 5,24$ & 45,6 & 75,5 & $50,84 \pm 3,69$ & 41,8 & 63,5 & $<0,01$ \\
\hline $12 \mathrm{~h} 45$ às $13 \mathrm{~h} 14$ & $59,39 \pm 4,97$ & 45,5 & 77,0 & $51,64 \pm 4,93$ & 36,5 & 64,8 & $<0,01$ \\
\hline 23h às 23h29 & $52,65 \pm 5,64$ & 34,8 & 69,9 & $46,51 \pm 5,49$ & 31,1 & 58,9 & $<0,01$ \\
\hline
\end{tabular}

$d B=$ Decibéis; $D P=$ Desvio padrão

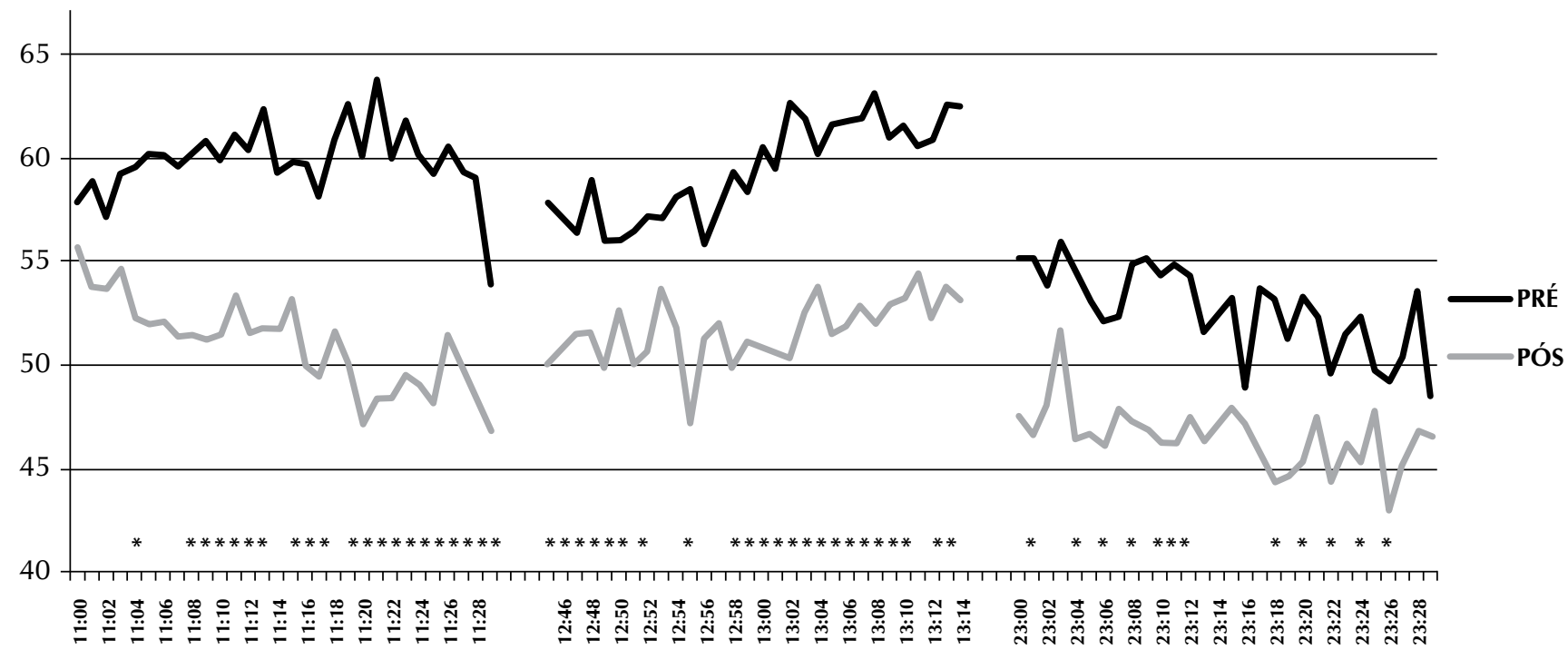

*Estatisticamente significante (Wilcoxon signed rank test)

Gráfico 1 - Intensidade de pressão sonora (em decibéis) de acordo com o horário (medianas), antes e após a intervenção educacional. 
Tabela 2 - Etiologia dos ruídos e sua intensidade, em valores médios.

\begin{tabular}{ll}
\hline Etiologia & $\begin{array}{c}\text { Nível de pressão } \\
\text { sonora [dB(A)] }\end{array}$ \\
\hline Equipamentos & 59 \\
\hline Alarme da oximetria de pulso & 59 \\
Monitor cardíaco & 57 \\
Alarme do ventilador mecânico & 57 \\
Aspiração endotraqueal & 48 \\
Alarme da bomba de infusão & \\
\hline Operacionais e comunicativos & 77 \\
\hline Abrir cortinas & 70 \\
Transporte de pacientes (ao lado) & 69 \\
Carrinho de metal & 68 \\
Lixeiro & 65 \\
Telefone tocando & 62 \\
Bater prontuário na mesa & 62 \\
Conversas entre profissionais da equipe & 61 \\
Bater gavetas & \\
\hline
\end{tabular}

$d B(A)$ : Decibéis níveis de audição

As médias das medidas nos três horários $(11 \mathrm{~h}$ às $11 \mathrm{~h} 29$, $12 \mathrm{~h} 45$ às $13 \mathrm{~h} 14$ e das $23 \mathrm{~h}$ às $23 \mathrm{~h} 29$ ) são indicadas na Tabela 1. Em ambos os momentos da coleta (pré e pós intervenção) os valores mais baixos se concentraram no horário noturno.

Quando se comparam as medianas dos dados da primeira com a última coleta, em 57 dos 90 horários houve redução com diferença estatisticamente significante (gráfico 1). A redução foi mais importante no horário de 11 h às 11 h29 $(15,0 \%)$ e menor no período noturno $(11,7 \%)$.

A intervenção educativa (segunda etapa da pesquisa) foi realizada por meio de palestra e distribuição de material educativo. No total participaram da intervenção educativa 35 profissionais, sendo médicos, enfermeiros, técnicos de enfermagem, zeladoras, secretárias e nutricionista que trabalham dentro da UTI do hospital. A adesão às aulas foi de cerca de $30,5 \%$ da equipe de enfermagem e 38,9\% dos médicos atuantes na UTI. No entanto, a totalidade dos profissionais da equipe foi abordada através de pequenas reuniões e distribuição de folders.

Em relação à origem dos ruídos encontrados, foi observado que os maiores níveis de pressão sonora foram gerados pela equipe que atua na UTI e não pelos equipamentos de monitoramento, como podemos observar na Tabela 2.

\section{DISCUSSÃO}

A busca por um ambiente menos estressante para a equipe e os pacientes e familiares é uma das prioridades na terapia intensiva atual ${ }^{(11)}$. Embora haja dados conflitantes em relação aos familiares ${ }^{(12)}$, tanto pacientes quanto a equipe claramente são influenciados negativamente pelo ruído ambiental e suas consequências ${ }^{(13)}$.

A UTI apresenta vários elementos que contribuem para altos índices de ruído ambiental: os equipamentos de terapêutica e monitorização, particularmente os alarmes e alertas sonoros; computadores, impressoras, aparelho de fax e telefones, movimentação de móveis, carrinho de banho, o diálogo dos profissionais entre si e com os pacientes, etc. Além disso, o tipo de ambiente em si (fechado e com paredes que refletem o som) pode aumentar o nível e consequências do ruído ${ }^{(14)}$. No entanto, o principal responsável pelo ruído na UTI pode ser a própria equipe ${ }^{(15)}$.

A Associação Brasileira de Normas Técnicas ${ }^{(16)}$ recomenda que, em áreas hospitalares, o máximo de intensidade sonora seja de 35 a $45 \mathrm{~dB}(\mathrm{~A})$. Para a OMS, os níveis de som dentro da UTI não devem ser maiores que $35 \mathrm{db}(\mathrm{A})^{(17)}$. Neste estudo, à semelhança de anteriores ${ }^{(7,13)}$, os valores mensurados excederam, em todos os horários, estes limites máximos, mesmo no período noturno.

Mudanças estruturais, como alterações ergonômicas, revestimento acústico do ambiente, e mesmo uso de protetores auditivos, mostraram reduzir o nível de ruído ambiental ou sua percepção pelos pacientes ${ }^{(10)}$. Contudo, o fator humano pode ser importante na gênese e manutenção do ambiente estressante na UTI, particularmente em relação ao ruído(2). Mais importante, tem-se demonstrado que frequentemente a equipe não tem conhecimento da importância do ruído no ambiente, bem como ignora seus mecanismos de etiopatogenia ${ }^{(18)}$. Assim, a educação continuada pode ter um importante papel na redução do ruído na $\mathrm{UTI}^{(10)}$, como o atual estudo mostra.

A intervenção educativa junto à equipe tem sido demonstrado ser eficiente na melhora da eficiência em distintas situações como diagnóstico e manejo da sepse, prevenção de pneumonia e prevenção de úlceras de pressão, entre outros ${ }^{(19)}$.

Estratégias que envolvam a participação dos membros da equipe, como dramatizações, são mais eficientes em aumentar a aderências a estratégias e bundles ${ }^{(20)}$. Portanto, esta metodologia utilizada neste estudo pode ter contribuído para os bons resultados de redução do ruído encontrados. Monsen \& Edel-Gustafsson ${ }^{(9)}$ mostraram melhora do padrão do sono em pacientes de UTI neurológica após um programa de modificação comportamental com a equipe, com duração de duas semanas.

Os valores de pressão sonora encontrados neste estudo foram particularmente altos, principalmente no período pré-intervenção. Mesmo após a intervenção educativa, a maioria das mensurações ultrapassou $40 \mathrm{~dB}$.

Sabe-se que os níveis de ruído entre $55 \mathrm{~dB}$ a $65 \mathrm{~dB}$ podem produzir excitações nervosas e estresse, tornando os pacientes mais sensíveis à dor, fazendo com que a quantidade de remédios seja aumentada ou seu uso prolongado. Níveis de ruído acima de $65 \mathrm{~dB}$ podem levar as pessoas ao infarto, arteriosclerose, infecção e osteoporose. Acima de $85 \mathrm{~dB}$, por tempo prolongado, pode causar alterações fisiológicas, como a perda auditiva pela degeneração celular da cóclea, distúrbios neuropsíquico, risco de hipertensão arterial, diminuição da 
resistência física e da concentração mental ${ }^{(6)}$. Isto é particularmente importante neste estudo, já que a mensuração foi feita à cabeceira do leito do paciente, ou seja, simulando o que cada paciente sente. Por outro lado, o impacto nos profissionais de saúde pode ter sido mesmo subestimado, já que alguns ruídos freqüentes (como tampas batendo, telefone tocando e conversas altas) são em geral mais longe dos pacientes.

Durante a realização desta pesquisa foi observado que o ruído presente na UTI tem sua origem diversificada, vindo de equipamentos e seus respectivos alarmes, de causas operacionais, como abrir lixeiro, empurrar carrinho de metal ou de banho, abrir e fechar gavetas, portas e cortinas, telefones, fax, computadores, impressoras, celulares, entres outros e causas comunicativas, como conversas entre funcionários e pacientes. A maior fonte de pressão sonora foi causada por fatores operacionais e comunicativos.

Os horários de maior intensidade de ruído foram relacionados à visita familiar e passagem de plantão; por outro lado, o horário noturno apresentou menor nível de ruído do que as medidas diurnas. Estes achados são similares a encontrados na literatura ${ }^{(7,13)}$.

Não foi possível verificar, comparando-se os períodos pré e pós-intervenção educativa, quais fatores foram mais reduzidos e contribuíram para um menor ruído. Contudo, o fato de a redução ter sido mais intensa nos horários de maior movimento de pessoas (durante o dia) sugere que a aderência da equipe deve ter tido um papel preponderante nesta redução do nível de ruído ambiental.

Este estudo tem várias limitações, algumas das quais já expressas acima. A coleta de pressão sonora foi feita apenas em momentos específicos, o que poderia ter subestimado o ruído no ambiente. No entanto, como já referido, os horários escolhidos procuraram refletir os momentos mais significativos: visita de familiares, troca de plantão (que notoriamente são os horários de maior nível de ruído) e horário noturno (que corresponde ao momento de menor agitação dentro da unidade) ${ }^{(7-8)}$.

O tempo decorrido entre a intervenção educativa e a segunda medida foi curto (cerca de duas semanas). Além disso, o período de intervenção em si foi curto. Portanto, uma intervenção mais prolongada ou contínua poderia ter efeitos mais duradouros. Também, não foi avaliado se esta redução do ruído foi persistente após uma única intervenção educativa.

Um outra abordagem poderia ser da mensuração das consequências do ruído ambiental nos pacientes e/ou equipe, p.ex. incidência de delirium, burnout ou PTSD, e o impacto de intervenções educativas. Porém, o desenho deste estudo dirigiu-se à medida do ruído em si, e não a consequências psicológicas.

\section{CONCLUSÃO}

Os níveis de pressão sonora encontrados na presente pesquisa estão acima do recomendado pelas normas técnicas da ABNT e pela OMS. As principais fontes sonoras de ruídos foram os fatores operacionais e comunicativos como diálogo entre profissionais e passagem de plantão, sendo, portanto, passíveis de redução, mesmo sem mudanças drásticas de engenharia.

A intervenção educativa junto à equipe multiprofissional, através de conscientização sobre a importância e causas geradoras de ruído, mostrou ser um importante instrumento para redução do nível de pressão sonora. Estudos avaliando a eficiência de intervenções mais duradouras poderiam mostrar o impacto em mantermos um ambiente mais saudável no manejo do paciente criticamente enfermo.

\section{REFERÊNCIAS}

1. Pattison N. Psychological implications of admission to critical care. Br J Nurs 2005;14(13): 708-14.

2. Dawson D. The problem of noise and the solution of sound? Intens Crit Care Nurs 2005;21: 197-8.

3. Vinodhkumaradithyaa A, Srinivasan M, Ananthalakshmi I, Kumar D, Rajasekhar RJ, Daniel T, Hirumalaikolundusubramanian $\mathrm{P}$. Noise levels in a tertiary care hospital. Noise Health 2008;10(38): 11 .

4. Donchin Y, Seagull FJ. The hostile environment of the intensive care unit. Curr Opin Crit Care 2002;8:316-20.

5. Garcia ACE. Biofísica: física dos sons. São Paulo: Sarvier; 2002.

6. World Health Organization [homepage na internet] Occupational and community noise. [acesso em 13 jan 2011]. Disponível em: http://www.who.int/mediacentre/ factsheets/fs258/en/

7. Christensen M. Noise levels in a general intensive care unit: a descriptive study. Nurs Crit Care 2007;12(4):188-97.
8. Macedo IS, Mateus DC, Costa EM, Asprino AC, Lourenço EA. Noise assessment in intensive care units. Braz J Otorhinolaryngol 2009;75(6):844-6 .

9. Monsen MG, Edell-Gustafsson UM. Noise and sleep disturbance factors before and after implementation of a behavioural modification programme. Intensive Crit Care Nurs 2005; 21(4):208-19.

10. Xie H, Kang J, Mills GH. Clinical review: The impact of noise on patients' sleep and the effectiveness of noise reduction strategies in intensive care units. Crit Care 2009;13:208.

11. Alameddine $M$, Dainty KN, Deber R, Sibbald WJ. The intensive care unit work environment: current challenges and recommendations for the future . J Crit Care 2009;24:243-8 .

12. Costa JB, Felicetti CR, Costa CRLM, Miglioranza DC, Osaku EF, Versa GLGS, et al. Stressful factors for families of critically ill patients of the intensive care unit. J Bras Psiquiatr 2010; 59(3):182-189 . 
13. Ryherd EE, Waye KP, Ljungkvist L. Characterizing noise and perceived work environment in a neurological intensive care unit. J Acoust Soc Am 2008;123(2):747-56 .

14. Ulrich R, Quan X, Zimring C, Joseph A, Choudhary R . [Homepage na internet] The role of the physical environment in the hospital of the 21st century: A once-in-a-lifetime opportunity. Report to the Center for Health Design for the Designing the $21^{\text {st }}$ Century Hospital Project. [acesso em 13 jan 2011]. Disponível em:http://www.rwjf.org/files/ publications/other/RoleofthePhysicalEnvironment.pdf

15. Kahn D, Cook T, Carlisle C, Nelson D, Kramer N, Millman R. Identification and modification of environmental noise in an ICU setting. Chest 1998;114(2):535-40.

16. Associação Brasileira de Normas Técnicas. NBR 1052/1987: Níveis de ruído para conforto acústico
- Procedimento - Versão corrigida 1992. Rio de Janeiro: ABNT; 1987.

17. Berglund B, Lindvall T, Schwela DH. [homepage na internet] Guidelines for community noise. Protection of the human environment. World Health Organization; 1999 [Acesso em 13 jan 2011]. Disponível em: http://www. who.int/docstore/peh/noise/guidelines2.html.

18. Christensen M. What knowledge do ICU nurses have with regard to the effects of noise exposure in the Intensive Care Unit? Intensive Crit Care Nurs 2005;21(4):199-207.

19. Skees J. Continuing education: a bridge to excellence in critical care nursing. Crit Care Nurs Q 2010;33(2):104-16.

20. Silva GM, Seiffert OMLB . Educação continuada em enfermagem: uma proposta metodológica. Rev Bras Enferm 2009;62(3):362-6. 